\title{
Extra-anatomic aortic bypass combined with Bentall procedure for a complex coarctation and aortic aneurysm
}

\author{
Yongqiang Jin ${ }^{1}$, Rui Liu ${ }^{1}$, guoliang chen ${ }^{1}$, Xiaoya Zhang ${ }^{1}$, and Qingyu $\mathrm{Wu}^{1}$ \\ ${ }^{1}$ Beijng Huaxin Hospital First Hospital of Tsinghua University
}

February 5, 2021

\begin{abstract}
Surgical treatment of complex coarctation is still a challenge. We performed extra-anatomic aortic bypass and Bentall procedure for a 10-year old boy diagnosed with critical aortic coarctation, aortic aneurysm, and severe aortic valve insufficiency who previously received Switch procedure. The patient is alive and well 52 months postoperatively with a satisfactory result. Extra-anatomic aortic bypass is a safe and effective procedure for patients with complex coarctation.
\end{abstract}

\section{Introduction}

Surgical treatment of complex coarctation is still a challenge, extra-anatomic aortic bypass maybe a safe and effective procedure. Herein, we describe a 10-year old boy diagnosed with critical aortic coarctation, aortic aneurysm, and severe aortic valve insufficiency who previously received Switch procedure, undergoing median sternotomy for extra-anatomic aortic bypass and combined Bentall procedure with a satisfactory result.

\section{Patient profile}

A 10-year old boy, diagnosed with right ventricular double outlet (Taussig-Bing type) at 2 months after birth, received arterial switch procedure with ventricular septal defect repair via the median sternotomy. He was admitted to our hospital with a 1-month history of refractory hypertension. His body weight was 40kg. Physical examination revealed an arm-leg systolic pressure differential of $70 \mathrm{mmHg}$. His femoral pulses were faint and pedal pulses were absent. Diastolic murmur along the left sternal border accompanied with wide pulse pressure and peripheral vessel sighs were observed. His chest radiograph was shown inFigure 1. Computed tomography revealed that the aorta was anterior to the left, and the pulmonary artery was posterior to the right. There was a critical coarctation of the aorta (COA) distal to the left subclavian artery with a luminal diameter of less than $5 \mathrm{~mm}$ and extensive collateral thoracic vasculature. Notably, the aortic root was significantly dilated to $65 \mathrm{~mm}$ measured on 3-dimensional cross-sectional imaging, as present in Figure 2 . Transthoracic echocardiography demonstrated a severe insufficiency of tricuspid aortic valve and a significantly dilated aortic annulus. His trans-coarctation pressure gradient was up to $76 \mathrm{mmHg}$. Written informed consent with the approval of our institutional review

board was obtained from the patient's family before performing the

procedure.

Extra-anatomic ascending-descending aortic bypass and Bentall procedure was performed with median sternotomy in a single-stage operation. Cardiopulmonary bypass (CPB) was instituted via the distal portal of the ascending aorta and both caval cannulation. Intraoperative transesophageal echocardiography and inspection of aortic root revealed that one leaflet of the tricuspid aortic valve underdeveloped with significant insufficiency, not allowing for valve-sparing root replacement. A $23 \mathrm{~mm}$ mechanical valve with aortic tube 
graft was employed to replace the aortic valve, aortic root, and proximal portion of the ascending aorta. Coronary ostia were re-implanted into the neo-aortic graft. Before the root portion of the procedure was performed, a 20-mm woven Dacron graft was anastomosed to the descending thoracic aorta via a posterior pericardial approach. After completion of the Bentall procedure, the descending graft was positioned around the left border of heart and anastomosed to the neo-ascending aortic graft. The patient was weaned from CPB uneventfully. Total aortic cross-clamp time was 184 minutes and CPB time was 329 minutes. Monitoring of radial and femoral arterial line pressures demonstrated no residual arm-leg pressure gradient.

The patient was discharged without any major complications. Post-operative transesophageal echocardiography revealed functional prosthetic valve and preserved left ventricular systolic function. Postoperative chest radiograph and computed tomography demonstrated that the extra-anatomic aortic bypass graft ran along with the left border of the heart and was patency (Figure 3\&4 ). During 52-months of follow-up, there was a significant reduction in mean upper-extremity blood pressure after operation without medication.

\section{Discussion}

COA is a common congenital cardiovascular malformation. It has been reported in the literature that $90 \%$ patients of untreated coarctation die within 50 years, usually due to heart failure, coronary artery disease, aortic dissection or rupture, infective endocarditis, or cerebral hemorrhage [1].COA is usually isolated, but it may be complicated with other major concomitant intra-cardiac anomalies. Patients with Taussig-Bing deformity are prone to accompany with coarctation and should be managed in surgical correction in a singlestage operation. In this case, coarctation had been undiagnosed before the previous operation until that the giant aortic root aneurysm and significant aortic valve insufficiency has developed.

The most common incision for repair of coarctation is left thoracotomy. However, for this patient, ascendingdescending aortic bypass which was first reported by Edie and colleagues may be the best and safest procedure [2]. Firstly, it can be done through a median sternotomy allows for concomitant aortic valve and root procedures in a single-stage operation. Secondly, extensive dissection can be avoided so that the risks of massive hemorrhage, injury of phrenic, recurrent laryngeal nerve and spinal cord ischemia would be limited [3]. Furthermore, although the patient was only 10-year old, the body size was approximate to an adult's, $20 \mathrm{~mm}$ graft can provide adequate flow during adulthood. What is different from previous surgeries is that the artificial graft of this patient was placed on the left side of the heart. In order to minimally compress the left ventricle, and for the consideration of future growth, we placed the artificial graft in the left thoracic cavity with sufficient length. The difference was mainly because that the ascending aorta was posterior to the left after Switch procedure.

Aortic dilation in COA is well documented, and this disorder is associated with ascending aortic medial degeneration. In addition, aortic dilation is a well-known complication after the switch procedure. Aortic root and aortic valve surgery after Switch procedure occur in approximately $2 \%$ of patients at 10 years [4]. These two reasons contributed to the aortic root aneurysm and aortic valve insufficiency. At operation, it was found that the aortic valve could not be repaired, so Bentall procedure which involves replacement of aortic valve and ascending aorta was the optimal strategy.

Funding : None.

Conflict of Interest : The authors declare that there are no conflict of interests.

Data Availability Statement : All data used during the study appear in the submitted article.

\section{References}

1. Jenkins NP, Ward C. Coarctation of the aorta: natural history and outcome after surgical treatment. QJM 1999; 92:365-371.

2. Wu Q, Chen X, Li H, Wang L, Xue H. Ascending-to-descending aortic bypass via posterior pericardium for complex coarctation of aorta. J Card Surg 2009;24(2):167-169. 
3. Edie RN, Janani J, Attai LA, Malm JR, Robinson G. Bypass grafts for recurrent or complex coarctations of the aorta. Ann Thorac Surg 1975; 20:558-566.

4. Fricke TA, d'Udekem Y, Richardson M, Thuys C, Dronavalli M, Ramsay JM, Wheaton G, Grigg LE, Brizard CP, Konstantinov IE. Outcomes of the arterial switch operation for transposition of the great arteries: 25 years of experience. Ann Thorac Surg 2012; 94:139-145.

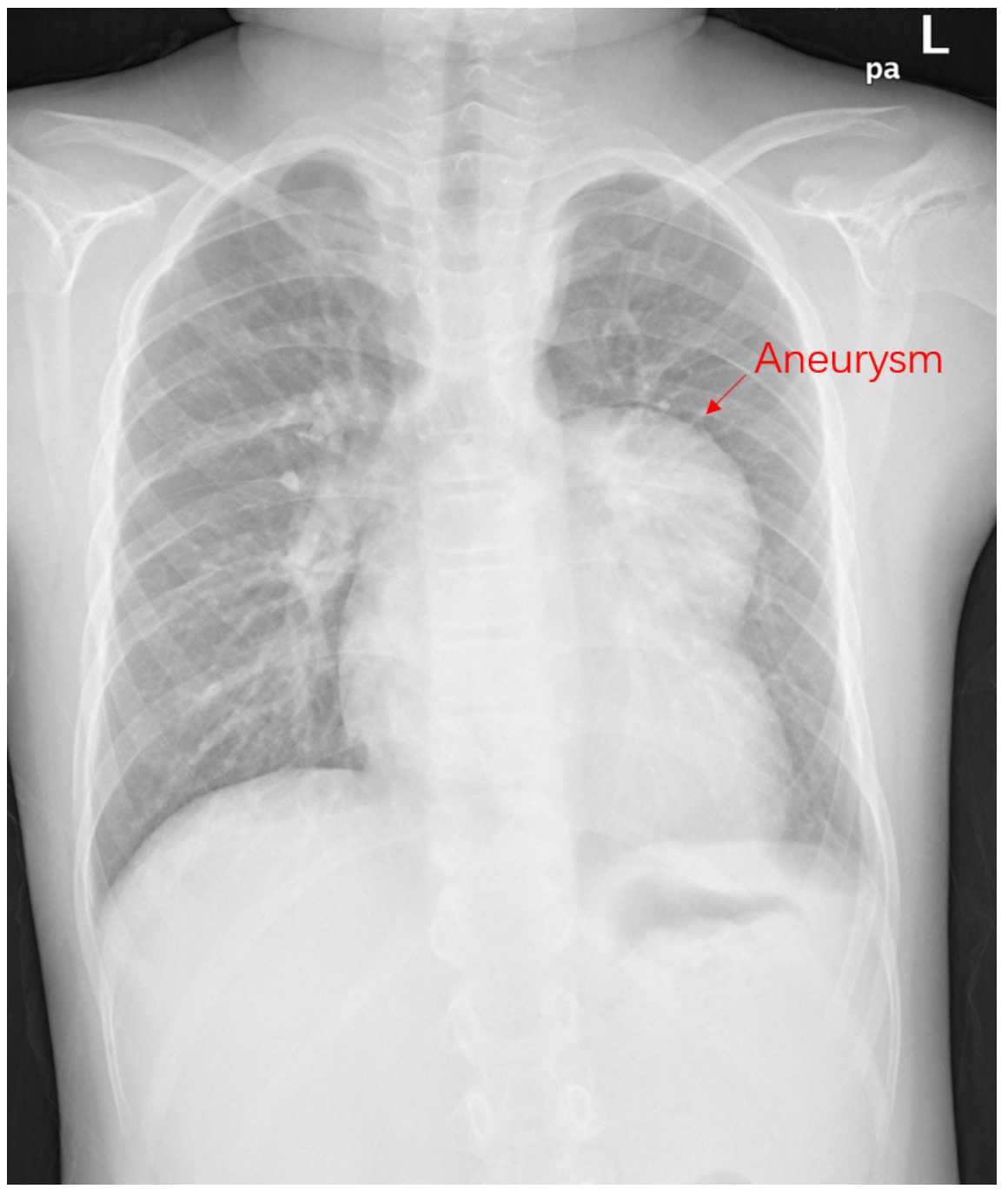




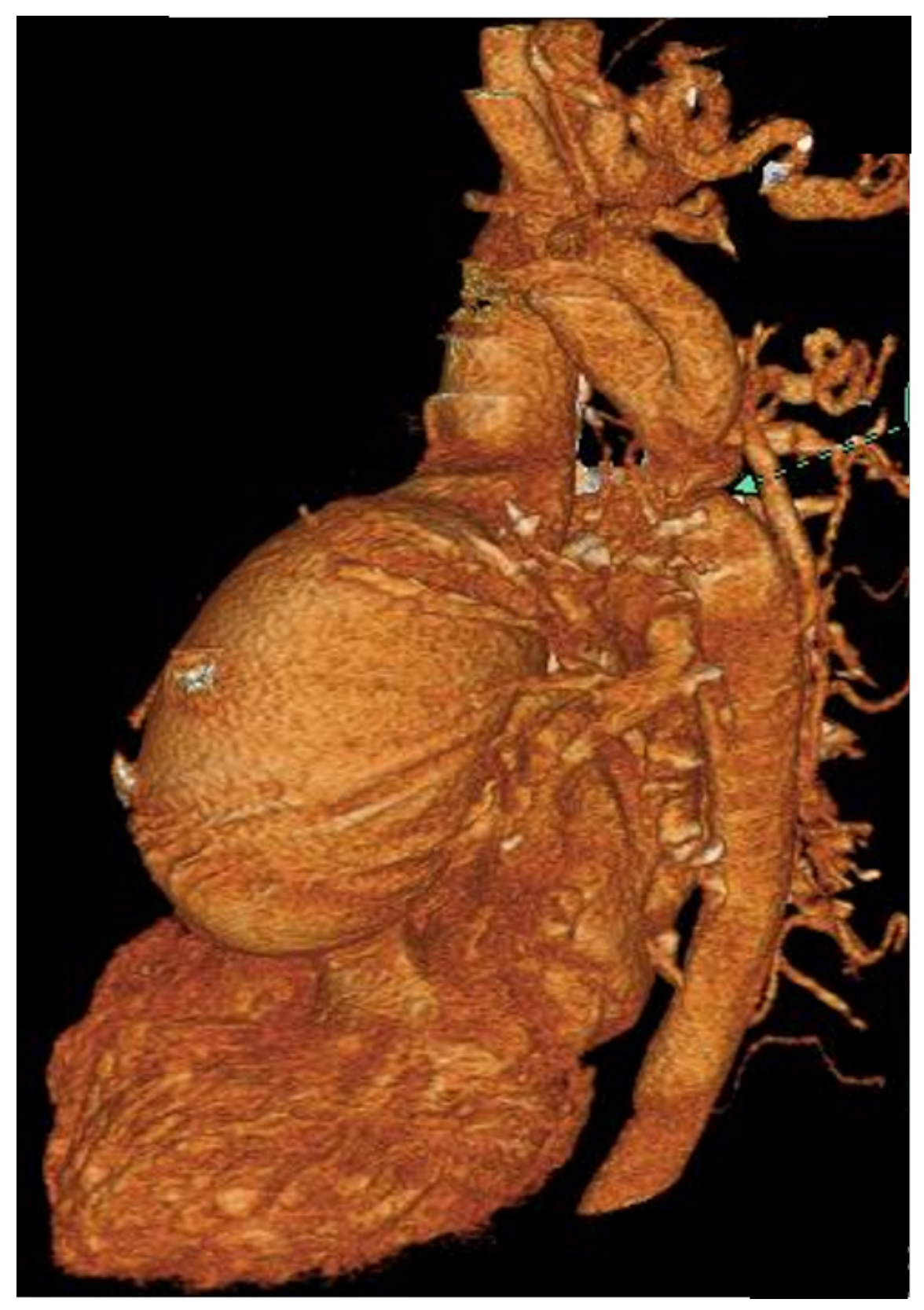




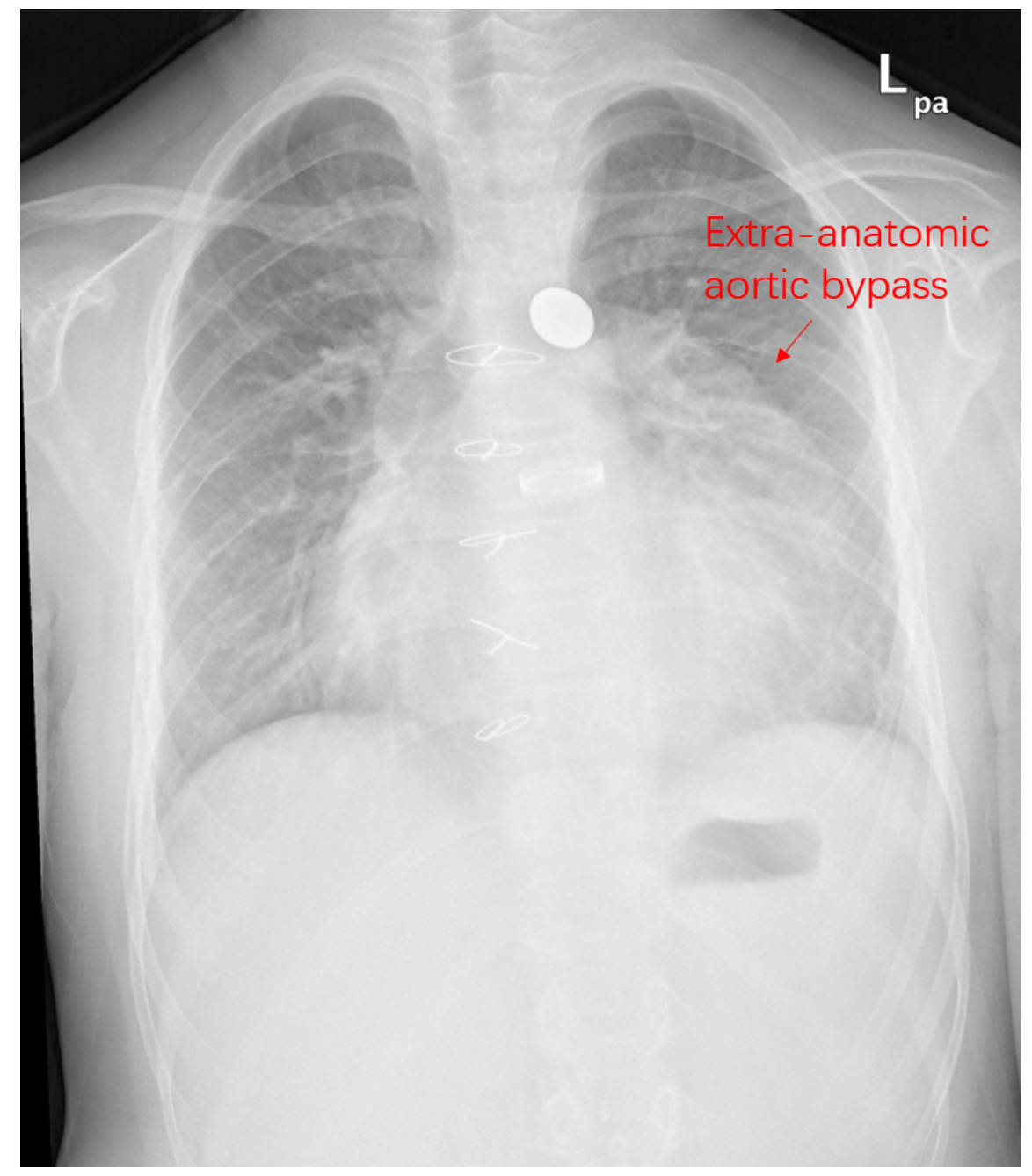




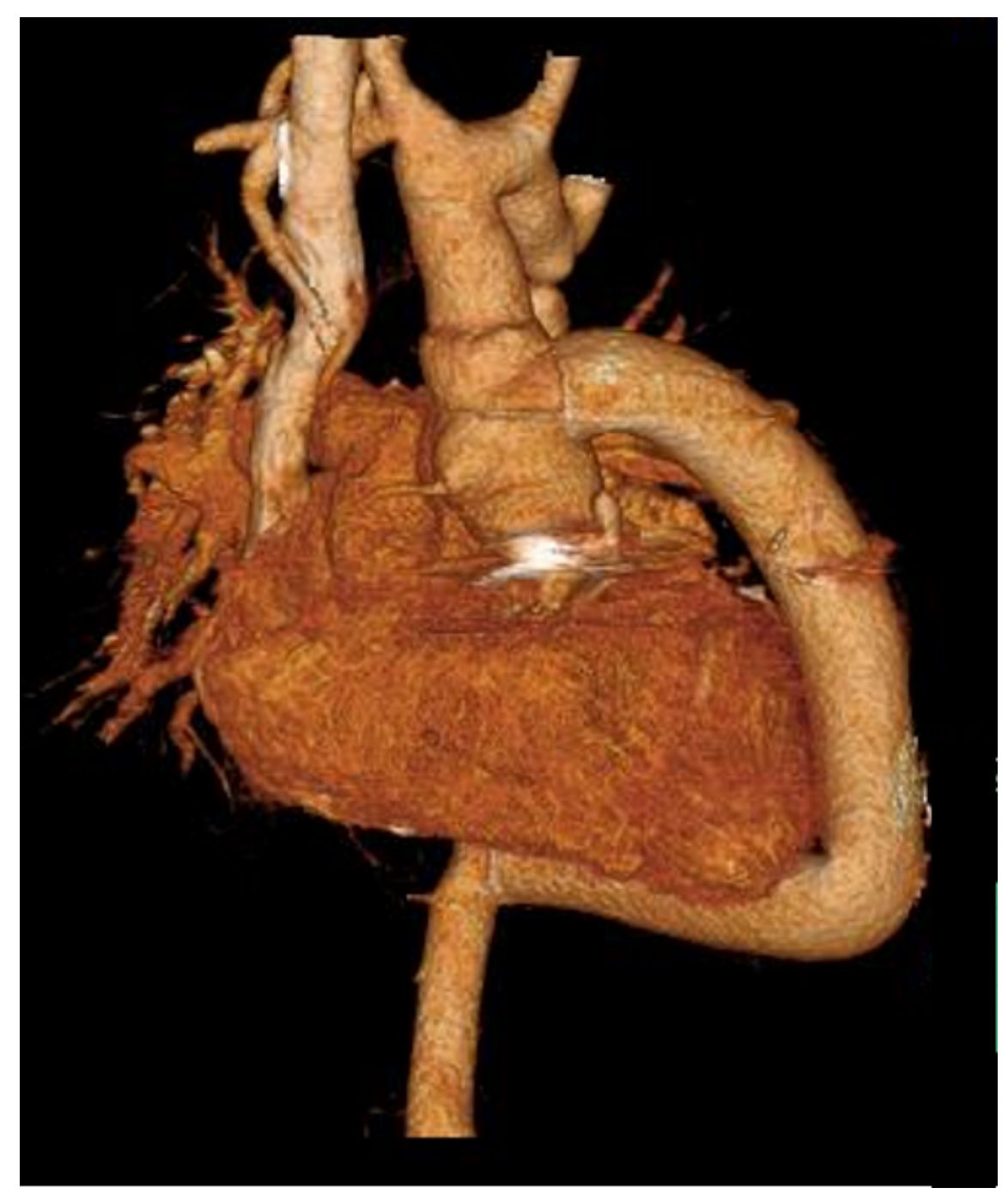

